\title{
La Evolución de la obra y el Modelo de Vittorio Guidano: Notas Histórico Biográficas
}

\author{
Vittorio Guidano: \\ The Evolution of His Work and Paradigm \\ Historical and Biographical Comments
}

Andrés Moltedo ${ }^{1}$

\section{Resumen}

En el presente artículo se revisa la evolución del pensamiento del psiquiatra y psicoterapeuta italiano Vittorio Guidano (1944-1999), considerado como uno de las figuras seminales del cognoscitivismo italiano, señalando algunos hitos epistemológicos e históricos que acontecieron de manera coetánea a su devenir teórico desde lo conductual a lo cognitivo estructural y, finalmente, post-racionalista. Asimismo se delinean de manera final, algunas líneas que han continuado el desarrollo del modelo.

Palabras clave: Vittorio Guidano, Epistemología, Psicología cognitiva, Post-racionalismo.

\begin{abstract}
The following article will review the evolution of thought in time of Italian psychiatrist and psychotherapist Vittorio Guidano (19441999), considered one of the founders of the Italian cognoscitivism. References will be made to some relevant epistemological and historical landmarks that took place simultaneously to his theoretical development that went from the behavioral to the cognitivestructural to finally reach a post-rationalist posture. Likewise, some final trails that have permitted a further development of the paradigm itself will be also explored.

Key words: Vittorio Guidano, Epistemology, Cognitive Psychology, Post-Rationalism.
\end{abstract}

Psicólogo, Escuela de Psicología Pontificia Universidad Católica de Valparaíso. andres.moltedo@ucv.cl 
La mia vita la definirei una sorta di 'Incompiuta' di Schubert, una sinfonia in due tempi, lasciata volutamente incompiuta Vittorio Guidano

\section{Introducción}

El estudio y el acercamiento a la obra de todo pensador siempre resulta una tarea compleja en varios sentidos. Existe una serie de cambios y de procesos tácitos internos que la persona jamás hizo (o no pudo hacer) explícitos. Se trata, en definitiva, de una "puntuación" o de un orden subjetivo realizado, buscando encontrar una continuidad y un cierto sentido en procesos que sólo son comprensibles en términos tácitos. Además, realizar una revisión histórica y bibliográfica de la obra de un autor, ya fallecido, es siempre una tarea difícil y, más aún, tratar de revelar los hilos teóricos y cambios epistemológicos que se entrelazan muchas veces de manera sutil.

Esta tarea es especialmente compleja cuando se trata de analizar la evolución de la obra de Vittorio Guidano², cuyos escritos han sido escasamente traducidos al español y resultan de difícil acceso. De una excepcional formación y cultura, para responder la pregunta respecto de la experiencia humana, utiliza las más diversas fuentes y disciplinas, y experimenta un proceso en su obra y en su quehacer terapéutico desde una inicial pragmática conductual y etológica, a una posterior búsqueda epistemológica y, finalmente, arriba a una inquietud de tipo ontológico.

A continuación, se pretende realizar un paralelo entre la evolución de la Psicología Cognitiva italiana en conjunto con la figura de Vittorio Guidano, y los cambios que realiza en el más conocido de sus conceptos: la Organización de Significado Personal.

2 Nacido en Roma el 4 de Agosto de 1944, se doctoró en Medicina y Cirugía en Universidad de Roma "La Sapienza" en 1969, obtiene su especialización en Neuropsiquiatría en la Universidad de Pisa en 1972. 


\section{Guidano estructuralista}

El primer centro de terapia cognitiva italiano ${ }^{3}$ fue fundado en 1978 , en la ciudad de Roma, por Guidano, Liotti, Reda, Chiari, Mancini y Gardner (entre otros), los cuales eran, en su mayoría, miembros del directorio de la Sociedad Italiana de Terapia Comportamental (SITC), la cual desde mediados de los años 70, había comenzado a extender su ámbito de influencias, especialmente gracias a la teoría que Vittorio Guidano y Giovanni Liotti se encontraban elaborando.

En 1979 ambos autores, en Elementi di Psicoterapia Comportamentale, manifestaron que buscaban conciliar la comprensión de los procesos y mecanismos psicológicos de la persona que sufre, con los modelos explicativos de la teoría de la ciencia. En dicho texto se hace evidente la influencia de autores como Popper (1974), Lorenz (1973), Bowlby (1969), así como de Miller, Galanter y Pribram (1960), entre otros. En este texto se aprecia el apego a una epistemología que se aparta de lo meramente conductual, y que es compatible tanto con la teoría de sistemas, como con la cibernética y con la teoría de la información. Dicha visión y epistemología de la psicoterapia será perfeccionada por ambos autores en conjunto en textos sucesivos $\mathrm{y}$, posteriormente, por dos caminos distintos ${ }^{4}$.

Como se ha dicho, ambos autores buscaban seguir en el ámbito de la psicología clínica las exigencias de una metodología que fuera objetivamente científica. Así, apegados al pensamiento de Popper, padre del racionalismo

\footnotetext{
Inicialmente bajo el nombre de "Centro de Psicoterapia Comportamental".
}

4 En palabras de Liotti (2006): "Después que PCDE fue publicado en 1983, Vittorio y yo seguimos trayectorias distintas. Vittorio continuó con la exploración de los procesos cognoscitivos que caracterizan la identidad personal, iniciada en este libro. A través de una originalísima conceptualización del sí mismo entendido como un proceso autopoyético complejo, Vittorio llegó a formular una versión de la psicoterapia que llamó "postracionalista”. Entre los trastornos psicopatológicos no tratados en este libro, la atención de Vittorio terminó concentrándose sobre la esquizofrenia. En toda su obra posterior a la publicación de PCDE, los resultados de la investigación empírica más reciente sobre el apego tienen una influencia relativamente modesta. Contrariamente, yo he continuado dedicándome básicamente a las aplicaciones clínicas de la investigación sobre el apego, que iban progresivamente extendiéndose al gran tema del apego desorganizado (aún no identificado en la época de la colaboración con Vittorio)" pág 15. 
crítico, buscan delinear una teoría que no se base exclusivamente en intuiciones subjetivas, sino que sea una teoría objetiva y discutible sobre las experiencias subjetivas con las que se trabaja en el ámbito clínico.

De esa manera, inicialmente Guidano y Liotti realizaron un estudio de las conductas de pacientes, para posteriormente examinar cuáles son las "estructuras cognitivas" que se encuentran a la base de la regulación de dichos patrones comportamentales. Así, mediante el estudio de secuencias complejas de conducta, y tras su análisis acorde a esquemas, buscaban apreciar y establecer relaciones entre dichos comportamientos con el ambiente en el que se realizan y, especialmente, con "las imágenes, expectativas, convicciones, relaciones entre procesos cognitivos y emociones, valoraciones y diálogos internos asociados a aquellas conductas" (Moltedo, inédito).

Tres años después, en 1981, en la introducción de "Cognitivismo e Psicoterapia” (Guidano y Reda, 1981) con la aparición del concepto de "Organización Cognitivo Comportamental"' dan un mayor énfasis y otorgan una mayor importancia a la función activa de los estados internos de un individuo en la elaboración del conocimiento.

Ese mismo año ${ }^{7}$ Chiari y Nuzzo organizaron en el Istituto di Genetica medica y Gemellologia de Roma el primer Congresso nazionale di terapia del comportamento e terapia cognitivo-comportamentale. Además de la elevada participación y convocatoria, acontecen dos grandes hitos:

La SITC comienza a expandirse fuera de su círculo romano creciendo a un nivel nacional.

Tras el trabajo del congreso, en la asamblea de socios se formalizó oficial y unánimemente el pasaje al cognoscitivismo, con la consiguiente modificación del nombre de aquel momento de la Sociedad, agregando una

5 "Por estructura cognitiva hacen referencia a las reglas que actúan como elementos organizadores de pensamiento y que coordinan la evolución, la calidad y la dirección de un sistema de representación, dotándolo de estabilidad interna, volviéndolo un estilo representativo" (Moltedo, inédito).

6 Constituida por necesidades, expectativas y convicciones dentro de un sistema unitario con coherencia interna y relativamente estable.

$7 \quad 20$ y 21 de Junio de 1981. 
"C" al acrónimo que la definía, pasando a denominarse Sociedad Italiana de Terapia Comportamental y Cognitiva (SITCC) (Chiari y Nuzzo, 1982).

Como queda de manifiesto a fines de la década de los setenta y comienzo de los ochenta, se hace patente la necesidad de un cambio de paradigma, visto como necesario para el crecimiento y el desarrollo de la psicoterapia cognitiva a nivel mundial (Semerari, 1998, 2000). Pero además de lo anterior, existía la convicción de no ser meros espectadores del cambio, sino más bien de poder contribuir a aquel, asumiendo un primer plano en este proceso de revolución teórica.

Dentro del cognoscitivismo mundial, había sido publicado en Estados Unidos, un año antes, Psychotherapy and the structure of personal revolutions (Mahoney, 1980), quien establecía una similitud entre los procesos de cambio psicoterapéuticos y la perspectiva epistemológica de Kuhn (1962). Se trata de un texto cuya lectura vino a confirmar los argumentos en pro del cambio epistemológico, y en donde el norteamericano establecía una serie de observaciones y críticas teóricas respecto de la terapia cognitivo-comportamental, que pretendían evidenciar la necesidad de una emancipación definitiva y de una clara diferenciación del cognoscitivismo respecto del conductismo.

Ese mismo año, Mahoney asistió a la Universidad de Roma, donde dictó una conferencia respecto del cambio ${ }^{8}$ inducido por las intervenciones terapéuticas. En dicha instancia expuso su idea, ya delineada en el párrafo anterior, del paralelo existente entre la "estructura" que sostiene el progreso científico y los mecanismos que gobiernan las modificaciones psíquicas de los pacientes en tratamiento.

Así, gracias a la beca Fulbright, Mahoney tomó contacto con la obra de Guidano y Liotti, quienes habían delineado una perspectiva similar a la suya para describir los procesos implicados en las variaciones de los significados personales, pero tomando como punto de partida la visión epistemológica de Imre Lakatos.

8 El cambio es un tema central en el pensamiento de Mahoney, siendo su libro Human change processes: the scientific foundations of psychotherapy (1991) un verdadero clásico mundial. 
Ante la proposición de Mahoney de editar Elementi di Psicoterapia Comportamentale ${ }^{9}$, en los Estados Unidos, Guidano y Liotti respondieron redactando un nuevo libro, acorde con los últimos desarrollos de su modelo explicativo de la psicopatología, que sería publicado en 1983 con el título de Cognitive processes and emotional disorders ${ }^{10}{ }^{11}$.

Esta obra que produjo el punto de inflexión de la evolución de la psicología cognitiva en Italia, y sentó las bases concretas de un modelo con una aproximación terapéutica completamente original, busca establecer una epistemología que no posea tintes asociacionistas ni reduccionistas, sino que genere "un modelo de la conducta humana que tenga implicaciones directas para la psicoterapia” (Zagmutt, 2006), que señala el paso del cognoscitivismo al constructivismo.

Debido a la excepcional cultura e inquietud intelectual que poseían Guidano y Liotti, es que en este libro confluyeron autores de las más variadas tradiciones y especialidades. Relacionaron los principios que gobiernan las fases del desarrollo y el "crecimiento" cognitivo elaborados por Piaget (1970; Piaget e Inhelder, 1966), con la teoría del apego y de los vínculos afectivos de Bowlby $(1969,1973,1980)$ y construyen un modelo de desarrollo psicológico unitario, sobre el cual fundar su trabajo clínico. De esa manera, por ejemplo, los aportes del británico les proporcionaron el sustento para otorgarle a la esfera emotiva una importancia fundamental, ya que para Guidano y Liotti, acorde con Leventhal (1979) y su concepto de emocional schemata, las reacciones emotivas preceden la emergencia del lenguaje y surgen de la relación de apego, junto con ciertas características temperamentales, formando parte de la constitución de una identidad personal desde las primeras fases del desarrollo.

\footnotetext{
9 Basado en un estudio de 1977.

10 Evaluado por la Behavioral Science Book Services como uno de los mejores del año, fue presentado y catalogado como "selección principal".

11 No extrañará que sea el mismo Mahoney quien escriba el prefacio del libro, donde realizará un paralelo entre los cambios terapéuticos y los cambios de paradigmas que caracterizan cada revolución científica; destacará, además, a este trabajo como un modelo heurístico de la organización del conocimiento personal y de su cambio.
} 
Guidano y Liotti, además, tomaron como punto de partida una visión evolucionista del conocimiento, basándose en las premisas epistemológicas de Popper $(1963,1972)$, en la epistemología evolutiva de Campbell (1974) y Lorenz (1973), y en la teoría motora de la mente desarrollada por Weimer (1977), para concebir al conocimiento como el producto de un sistema de construcción activa de la mente, negando el origen sensorial del conocimiento, así como la pasividad del organismo que conoce. Sin embargo, aún mantendría un tinte representacional, donde el papel del observador sería, principalmente, el de seleccionar una serie de estímulos intrínsecamente informativos.

Es el individuo el que evoluciona adaptándose al ambiente, organizando los procesos cognitivos propios de una manera activa, que influyen en la formación activa de expectativas, de hipótesis y de teorías que guían, a su vez, tanto la acción como la percepción, construyendo el conocimiento individual del mundo. En esta particular visión de la manera como se estructura el sistema de conocimiento del ser humano en su relación con el ambiente, la persona será el agente regulador central de la organización del conocimiento, cuya estructura intrapsíquica será descrita de acuerdo a la visión epistemológica de Lakatos (1974), y cuyos procesos tácitos y explícitos de la elaboración de la información lo serán de acuerdo a Polanyi (1966).

Dichos sustentos teóricos, junto con aportes provenientes de la etología y de la neuropsicología, delinearon el concepto de Organización Cognitiva ${ }^{12}$ que constituye otra contribución teórica original y distintiva de la escuela italiana, y que en dicho texto recibe por primera vez un trato sistemático, bajo la forma de "organización de la estructura cognitiva personal”. Estas organizaciones son descritas como estructuras con una identidad personal con dos ejes, la actitud hacia sí mismo (eje interno) y la actitud hacia la realidad (eje externo), siendo el apego la matriz que permite comprender cómo se construyen ambos ejes de conocimiento.

12 De forma similar al sistema de creencias de Meichenbaum, las estructuras cognitivas se organizarían como una teoría científica, de acuerdo a una estructura organizada que es capaz de construir, según el modelo de Lakatos, tanto una noción del mundo como un programa de investigación. 
Guidano y Liotti utilizaron la teoría de Bowlby (1969) como base y punto de partida para el establecimiento de los criterios que emplearon para distinguir a las organizaciones cognitivas, e hipotetizar que el origen de las diferencias individuales se halla en los diversos itinerarios de desarrollo de acuerdo a lo observado en un estudio etiopatogénico, en el cual llegaron a describir los aspectos estructurales, las características cognitivas, emotivas, comportamentales y los procesos estratégicos de tratamiento de cuatro modalidades de organización del conocimiento, las cuales presentarían "distorsiones cognitivas". Estas fueron agrupadas de acuerdo a los síndromes o diagnósticos principales que encontraron en su práctica clínica, es decir, organización fóbica (FOB), obsesiva (OBS), depresiva (DEP) y aquella específica de los trastornos alimenticios psicógenos (DAP).

En otras palabras, en el texto de 1983, tanto la psicopatología como los trastornos emocionales fueron reducidos casi exclusivamente a una patología del apego, y a la forma en que ella influye en la relación hacia sí mismo. De esa manera, Guidano y Liotti vincularon la patología vincular con el autoconocimiento, describiendo cuatro patrones anormales de vínculo y sus correspondientes distorsiones cognitivas. Si bien estas cuatro formas peculiares de articulación del conocimiento individual, utilizan como etiqueta una terminología derivada explícitamente de la nosografía psiquiátrica, no se refieren necesariamente a cuadros patológicos, sino que son utilizadas para indicar una modalidad del conocimiento que permite una adaptación funcional a la realidad (Guidano y Liotti, 1983).

Será la dinámica separación-apego la que conduzca los mecanismos de coordinación ${ }^{13}$ entre los distintos niveles de integración que definen la organización cognitiva. De acuerdo a los comportamientos de la figura de apego (que influirán tanto en las formas de aprendizaje como en los sistemas comportamentales del individuo) se establecerá una polaridad cognitiva (Guidano y Liotti, 1981), semejante a los esquemas imágenes/plano (Miller, Galanter y Pribram, 1960).

13 Action rules y Stopping rules. 
El modelo cognitivo estructural, en su desarrollo, modificó la vinculación tradicionalmente establecida entre las cogniciones y emociones en el cognoscitivismo, al insertar el primado de lo emotivo sobre lo cognitivo.

Como se ha podido apreciar, tras un proceso de larga evolución, la búsqueda epistemológica de un sentido para su quehacer terapéutico ha llevado a Guidano y a Liotti a alejarse del conductismo, pero también de los principios de la psicoterapia cognitiva tradicional. Este punto de llegada ha sido, a su vez, un nuevo punto de partida en dos sentidos: El primero se trata de la fundación del modelo cognitivo estructuralista (Cionini, 1991; Reda, 1992) que con el cambio de paradigma antes descrito caracteriza a la psicología cognitiva italiana de las últimas décadas del siglo XX. El segundo consiste en que, con posterioridad a este libro, ambos autores elaboraron en conjunto algunos artículos para luego emprender caminos por separado. Así, los temas tratados y delineados en este texto junto con Liotti, serían profundizados, reelaborados y complejizados en las sucesivas obras de Guidano.

\section{Guidano post-racionalista}

Después de años de un innovador trabajo en conjunto, en la segunda mitad de la década de los ochenta se produjo un distanciamiento y posterior separación entre Guidano y Liotti, respecto de la manera en la cual apreciaban ciertos tópicos y temáticas centrales del modelo que habían ido desarrollando, que se fue haciendo cada vez más clara, hasta desembocar en dos posiciones clínicas y teóricas distintas.

Además de un distanciamiento respecto del proceso de regulación del apego respecto de la figura vincular, sin lugar a dudas que la principal divergencia entre ambos consistió en el énfasis de orden epistemológico con el que sustentaban su enfoque clínico. Mientras que para Guidano la epistemología tiene una importancia fundamental y se encuentra imprescindiblemente ligada a la psicología, donde para él los mecanismos de crecimiento y cambio del conocimiento científico eran considerados como asimilables a aquellos mediante los cuales se incrementa el conocimiento individual (por ejemplo en la terapia), para Liotti la epistemología era un 
“instrumento útil a proveer conceptos que representasen los procesos a nivel psicológico más formalizados y definidos respecto de aquellos elaborados en el ámbito de la investigación experimental" (Moltedo, inédito).

Tras la separación de ambos autores, Guidano publicó en Estados Unidos sus dos grandes obras Complexity of the self (1987) y The Self in Process (1991), ambas publicadas en Italia al año siguiente de su versión en inglés. La perspectiva desarrollada en ambos textos es claramente de impronta post-moderna (Lyotard, 1979), acorde con el denominado "pensamiento débil" (Vattimo, 1983), incluso hasta llegar a acoger al constructivismo radical (Von Glaserfeld, 1984), en oposición a casi cualquier forma de empirismo.

Con el término "post-racionalismo", Guidano buscaba distinguir su concepción de los modelos clínicos de la psicoterapia cognitiva, fundados en la filosofía empirista y en el primado de la racionalidad, basados en una visión del conocimiento como representación correspondiente al mundo externo y real. Mientras que el post-racionalismo, en tanto constructivista, se basa en una idea del conocimiento como construcción de un ordenamiento tácito, donde los aspectos subjetivos de elaboración de la información asumen un rol fundamental.

En términos generales, para Guidano no nos encontramos como sujetos cognoscentes ante una realidad con un carácter de unívoca, objetiva y universal, sino ante un continuo fluir multidireccional y multiversal, ligado a procesos cognoscitivos individuales, con tantos "versos" posibles como observadores haya. Al no existir separación entre objeto y observador, la modalidad de construcción de la experiencia dice relación más con la viabilidad que con la corrección.

En su evolución, Guidano ha pasado ahora de una visión sistémicoprocesal, basada en la teoría general de sistemas (Von Bertalanffy, 1968) y la cibernética de primer (Wiener, 1948) y segundo (Von Foerster, 1981) orden, a una óptica que él ha definido como "post-racionalista", en la cual introdujo como líneas centrales entre las cuales desarrollar sus ideas clínicas, los conceptos de autopoiesis (Maturana y Varela, 1980), la concepción de apertura y cierre de un sistema vivo (Morin, 1977), la distinción entre orden físico y 
orden fenoménico (Von Hayek, 1952), y los estudios sobre los procesos irreversibles y del orden a través de las fluctuaciones (Prigogine, 1980, 1997).

Respecto de Cognitive Processes and Emotional Disorders (1983), en Complexity of the Self ${ }^{4}$ (1987), Guidano se distanció aún más del paradigma empírico-asociacionista al establecer como foco de atención de su modelo la epistemología de la complejidad, de acuerdo a la cual todas las formas de vida evolucionan asumiendo complejidades crecientes. Por esto es que resulta necesario considerar las capacidades de los organismos vivos para autoorganizarse y autodeterminarse, así como la plasticidad, flexibilidad y apertura de los procesos individuales de desarrollo, que están a la base del ordenamiento que hace de la realidad cada organismo.

De esa manera, las elaboraciones posteriores del constructo de organización de estructura cognitiva personal, efectuadas exclusivamente por Guidano, lo llevaron a la elaboración de un modelo que considera en el funcionamiento psicológico de las personas la articulación de procesos tácitos y explícitos del conocimiento en la construcción de significado (Arciero et al., 2005) tanto en la normalidad como en la patología, al enfatizar aspectos evolutivos y procesales.

Así, en el texto de 1987, Guidano concibió a la Organización Cognitiva Personal, como un concepto que engloba una "organización específica de los procesos de conocimiento personales que gradualmente emergen a lo largo del curso del desarrollo individual" (Guidano, 1987, p.91).

Para el autor, la naturaleza de los procesos cognitivos puede ser explicada tanto a través de la Teoría Motora de la Mente (Weimer, 1977) como del Modelo Holográfico de Elaboración de las Informaciones (Pribram, 1971), donde la Organización Cognitiva Personal se basa en las capacidades autorreferenciales del individuo que, de acuerdo a aquellas, ordena el flujo tácito de la experiencia. Se trata de un orden que coordina y regula cada aspecto de su vida conciente y del cual el organismo "extrae" su identidad personal, sentido de individualidad, unicidad, continuidad y

14 Elegido por Behavioral Science Book Services como la Selección Alternativa del año (Alternative Selection). 
estabilidad, que al ser autorreferencial, no depende ni de las propiedades ni de las cualidades intrínsecas del objeto ni del mundo externo.

De la misma forma que lo ha hecho en textos anteriores, en Complexity of the self (1987) la teoría del apego de Bowlby siguió manteniendo su carácter de paradigma integrador del proceso de desarrollo individual y de la organización de la realidad, de acuerdo con Guidano. Es en la ontogénesis que se efectúa al interior de la estructura provista por la relación vincular primaria de apego que se produce tanto la diferenciación emotiva como el desarrollo cognitivo.

Es en la reciprocidad ${ }^{15}$ que se experimenta con la figura de apego que se articulan los estados emotivos primarios, los cuales se van diferenciando en la ontogenia y poseen una función primaria respecto al desarrollo cognitivo (Ekman, 1972, 1984; Izard, 1977). Así, el sentido de sí del niño es primariamente de tipo afectivo, construyéndose en torno a esquemas emocionales básicos, los cuales son organizados de acuerdo a un conjunto de escenas estructuradas o scripts (Tomkins, 1978), constituyendo este guión, para Guidano, una herramienta integradora del sentir y el pensar, una cierta tonalidad emotiva del sentido de sí en relación a un evento, lo que sienta las bases para "la incorporación de los aspectos narrativos al modelo" (Zagmutt, 2006), cosa que Guidano efectuará en los últimos años de su vida.

Para Guidano (1987) la tendencia a mantener la coherencia sistémica será el mecanismo esencial que se encuentra a la base de los procesos autorregulatorios del sistema de conocimiento personal. Así, efectuando un nuevo desarrollo respecto de su modelo anterior, en este texto Guidano profundizó en la noción de self como un proceso de mantención de la continuidad, que se encuentra en una permanente reestructuración de Sí mismo, y no como un autoconcepto rígido. De esa forma, se distanció aún más de los enfoques cognitivos tradicionales al adoptar un enfoque más sistémico respecto a la organización del conocimiento, como ya se ha dicho, centrándose en la coherencia sistémica más que en la percepción de congruencia o incongruencia.

15 Especialmente de acuerdo al efecto de looking glass del proceso de identificación. 
Las cuatro organizaciones cognitivas personales, identificadas ya en el texto de 1983, fueron descritas con gran detalle respecto a los procesos generales de desarrollo, el desarrollo de la identidad, la resolución adolescente, la articulación de la experiencia emotiva, las estrategias de regulación (emotiva, cognitiva y comportamental), la coherencia sistémica, las características emotivas y cognitivas de la actitud hacia sí mismo y hacia la realidad, así como las dinámicas de las disfunciones cognitivas (surgidas ante discrepancias entre el nivel tácito y explícito frente a personas y eventos significativos).

Guidano, siguiendo el pensamiento de Marmor (1983) subrayó de manera expresa la independencia del constructo de Organización Cognitiva Personal respecto de las manifestaciones psicopatológicas. De esa manera propuso una psicopatología "que se hace cargo de la complejidad y la variabilidad de los desordenes emocionales mediante la elaboración de un modelo unitario, evolutivo y procesal" (Zagmutt, 2006), en el cual cualquier Organización Cognitiva Personal puede evolucionar a lo largo de un continuo (entre normalidad y psicosis ${ }^{16}$ ) de acuerdo a la calidad de la elaboración de la experiencia respecto a las dimensiones de abstracción/ concretud, flexibilidad y capacidad de integración.

Cuatro años después de Complexity of the Self (1987), publicó en Estados Unidos "The Self in Process" (1991), que se transformaría en el libro más difundido de Vittorio Guidano en español tras su publicación en este idioma en 1994.

En este libro nuevamente se puede apreciar un salto epistemológico, en el cual puso de manifiesto la imposibilidad para reducir la experiencia subjetiva a cualquier modelo de procesos cognitivos, aunque éstos sean complejos y se encuentren bien articulados.

Recurrió al pragmatismo norteamericano de Mead y James para explicar "la tensión intrínseca de la dinámica de la mismidad, es decir, un 'Yo' que experimenta y un 'Mí que continuamente reordena y explica a posteriori” (Oneto y Moltedo, 2002, p.88).

16 Se trata de un continuo donde se encuentran normalidad, neurosis y psicosis, a las que considera como estados contiguos, dinámicos, cambiantes y potencialmente reversibles. 
El "Yo", que actúa y experimenta, corresponde a la experiencia inmediata, a aquella irrefutable experiencia de ser en el mundo a la cual no es posible aplicar ningún criterio de verdad o falsedad. Es sólo a partir de un segundo momento en donde el Sí mismo es capaz de distinguir y ordenar esta experiencia. Es en ese instante que la experiencia adquiere consistencia y significado, en el "Mî". Esta dinámica de la mismidad interactúa en un proceso circular sin fin, donde la valoración sigue siempre al fluir de la praxis del vivir. Todo significado personal es, entonces, "el orden experiencial sobre el cual se percibe la continuidad y la coherencia del propio Sî" (Arciero et al., 2005, p. 25).

Sin embargo, paulatinamente se empezó a alejar de la noción de los procesos del self centrados en dicha circularidad al introducir la noción de "comprensión", entendida como un proceso interpretativo del vivir. A partir de lo cual, se empezó a apreciar un claro interés en el tema narrativo y hermenéutico de Ricoeur.

La autoorganización surge como condición necesaria para la mantención de la identidad y la integridad, a través de la adaptación y reestructuración constante ante las exigencias externas y las perturbaciones ambientales. Desde que nace, el ser humano busca constantemente darle significado tanto a las experiencias emotivas como a sus actos, que se desarrollan en un ambiente de vínculos intersubjetivos específicos, y que son compartidos a través de una dimensión simbólico-lingüística.

Es en la reciprocidad de la relación con la figura de apego que el Sí mismo se irá diferenciando, desplegándose de manera progresiva a través de constantes reestructuraciones producto de las perturbaciones de las experiencias emotivas vividas como poco comprensibles o poco controlables. El papel central del apego es "ampliado" con la noción de intersubjetividad, que le permite profundizar el papel del "otro" en la construcción de la identidad personal, el tema de la alteridad.

Como cada ser humano vive una experiencia inmediata reconocible y decodificable y, por lo tanto, explicable y significable, construye un mundo, pudiendo ser caracterizado por una Organización de Significado Personal, las cuales son conceptualizadas de acuerdo a una base epistemológica con una concepción autorreferencial del conocimiento, que "supera" al racionalismo crítico poppereano. Así, deja de lado cualquier 
vinculación con su pasado cognitivo-conductual, al desechar toda concepción representacional del conocimiento al evolucionar definitivamente a una autorreferencial. A esta altura de su desarrollo teórico, Guidano concebía al ser humano como alguien que no se encuentra ante una realidad intrínsecamente informativa, sino que ésta no es conocible en sí misma prescindiendo de un observador.

Mientras que en Cognitive Processes and Emotional Disorders (1983) la teoría del conocimiento era principalmente especulativa a partir de la aplicación de un modelo de desarrollo del conocimiento científico, en Complexity of the Self (1987) y, especialmente, en The Self in Process (1991) se trata de una teoría basada en las adquisiciones y logros científicos, especialmente en el ámbito de la biología y las ciencias de la complejidad.

Así, "alejándose cada vez más de una práctica de estampa cognitivoconductual tradicional, Guidano pone como fundamento de la Organización de Significado Personal el dominio emotivo, como aspecto que más que cualquier otro, da el sentido de unicidad personal y continuidad histórica que nos caracteriza como seres humanos" (Arciero et al., 2005, p. 26).

En el texto de 1991, las cuatro organizaciones, que se manifiestan en una configuración coherente de la actividad cognitiva, motora y, especialmente, emotiva-afectiva, representan cuatro categorías básicas o dimensiones de significado personal, que corresponden a las formas posibles de ordenamiento de la experiencia. Tienen su origen en una configuración vincular particular, y en un ensamblaje específico de escenas y escritos nucleares, que a lo largo del desarrollo de la existencia, serán expresión de una construcción subjetiva, de distinciones personales, dando lugar a diferentes reordenamientos del mundo y del fluir continuo de la experiencia propia.

El núcleo central de las Organizaciones de Significado Personal en The Self in Process está dado por los procesos tácitos. Éstos constituyen el mundo emotivo, desempeñan un rol primario en el proceso de conocimiento y mantienen tanto la atribución de significados como la coherencia de la organización. Respecto de este punto, quedó nuevamente en evidencia el distanciamiento de Guidano respecto de lo cognitivo-conductual, al establecer este núcleo en el dominio emotivo y no en los procesos cognitivos, ni las estrategias de solución de problemas, ni las representaciones de sí y del mundo. 
En lo referente a la psicopatología, en este libro las Organizaciones de Significado Personal carecen de vinculaciones, asociaciones y referencias directas o causales a una condición psicopatológica específica. Ya no son entendidas como una particular forma de disfunción cognitiva, sino como configuraciones particulares e individuales, un patrón emotivo característico recurrente y un modo específico de leer la propia experiencia interna. De esa manera, normalidad, neurosis y psicosis son consideradas como distintas dimensiones de la coherencia sistémica, diferenciándose y distinguiéndose principalmente por el grado de flexibilidad y generatividad con el cual articulan la experiencia. ${ }^{17}$

De acuerdo a esta evolución, es que junto a Giampiero Arciero y Maurizio Dodet, Guidano fundó en el año 1997 el Istituto di Psicologia e Psicoterapia Cognitiva Post-Razionalista de Roma (IPRA). Al año siguiente, presidió el comité científico del "VI Congreso Internacional sobre Constructivismo en Psicoterapia" (Siena del 2 al 5 de septiembre de 1998).

Guidano fallece de un infarto el 31 de Agosto de 1999 en Buenos Aires, a los pocos días de haber participado de un congreso en Chile. Jamás sintió que había concluido su obra, la cual, al igual que su visión del self, es un proceso que se fue complejizando y perfeccionando cada vez más, siendo cada vez más abarcativa y explicativa de la experiencia humana.

\section{Conclusiones}

La revisión que termina en estas líneas, nos ha llevado a recorrer la obra de Guidano y su entrelazamiento con la historia del cognoscitivismo clínico italiano del siglo XX.

Como delineábamos en la Introducción, el acercamiento a la obra de un pensador ya fallecido es una tarea compleja, tanto por los cambios y procesos tácitos internos que muchas veces no son explícitos, como por las "puntuaciones" realizadas al momento de revisar la obra por parte del lector.

\footnotetext{
17 Para una lectura más detallada del tema, remito al lector a Moltedo, A. (2004, 2007).
} 
Si bien pueden efectuarse lecturas del devenir histórico que acentúen distintos procesos u otras figuras del Cognoscitivismo Italiano, resulta innegable la influencia de Vittorio Guidano en dicho modelo y en su evolución, ya sea fundando el primer centro de terapia cognitiva de Italia, siendo director de la SITC, participando de su transformación a la SITCC, o liderando activamente a nivel mundial los cambios de paradigma que paulatinamente llevaron a variar el énfasis del conductismo hacia el cognoscitivismo y, finalmente, al constructivismo de la mano de figuras como Michael Mahoney.

Pero sin lugar a dudas, la mayor contribución de Guidano consistió en sentar las bases de un modelo con una aproximación terapéutica original, que al insertar el primado de lo emotivo sobre lo cognitivo, modificó la vinculación establecida tradicionalmente entre las cogniciones y emociones en el conductismo y en el cognoscitivismo tradicional, recuperando la emotividad y la subjetividad como tema central e hilo conductor. Aquella fue la dirección tomada desde la insatisfacción inicial con el paradigma comportamental, hasta los desarrollos más recientes, siempre de la mano de una profunda búsqueda de coherencia epistemológica.

Tras su muerte, se puede apreciar su legado en el desarrollo de diversos modelos que, basándose en esta inquietud y desarrollo teórico, buscan continuar este proceso sin fin. Dentro de los distintos núcleos de interés y de especificidad bien definidos, se pueden mencionar, entre otros: la depresión, la adolescencia y la filogénesis (Nardi, 2005); el evolucionismo (Liotti, 1994); el apego y la edad evolutiva (Lambruschi, 2004); la aplicación en los servicios públicos (Rezzonico y Lambruschi, 1996); el análisis conversacional (Lenzi y Bercelli, 1999) y el trabajo con dependencias (Panelli, 2003).

Otra de estas revisiones del modelo propuesto se entrelaza en un diálogo tanto con las neurociencias, como con la fenomenología hermenéutica, especialmente a partir del capítulo póstumo escrito junto con $\mathrm{Ar}$ ciero Experience, explanation and the quest for coherence del año 2000.

Así, las Organizaciones de Significado Personal y el modelo que las sustenta, mutan hacia el concepto de Estilos Personales, que no se centra exclusivamente sobre el significado sino también sobre la identidad. Esto se traduce en una apertura no sólo a un sentido personal sino también a 
hacer referencia a la situación, al modo de ser y a la experiencia de la que se habla. El paso de las Organizaciones de Significado Personal a los Estilos Personales, no se trata solamente de una reelaboración del concepto, sino que de un cambio epistemológico al interior del post-racionalismo.

\section{Bibliografía}

Arciero, G.; Gaetano, P.; Maselli, P.; Mazzola, V. (2005). Le organizzazioni di significato personale. En BARA, B. (Ed) Nuovo Manuale di Psicoterapia Cognitiva. Volume Secondo, Clínica. Bollati Boringhieri. Seconda edizione.

Arciero, G.; Guidano, V. (2000). Experience, explanation and the quest for coherence. En Neimeyer, R.A.; Raskin, J.D. (Eds) Constructions of Disorder. American Psychiatric Association.

Bowlby, J. (1969). Attachment and loss. Volume I. London: The Hogarth Press. [El vínculo afectivo, Paidós, 1993]

Bowlby, J. (1973). Attachment and loss. Volume II Separation. London: The Hogarth Press. [La separación afectiva, Paidós, 1993]

BowLby, J. (1977). The making and breaking of affectionals bonds: Etiology and psychopathology in the light of attachment theory. British Journal of Psychiatry. 130, 202-210 [Vínculos afectivos: formación, desarrollo y pérdida, Ed Morata, 1999].

Bowlby, J. (1980). Attachment and loss. Volume III Loss, sadness and depression. London: The Hogarth Press. [La pérdida afectiva, tristeza y depresión, Paidós, 1990]

Bowlby, J. (1988). A Secure Base. Clinical applications of Attachment Theory. London: Routledge. [Una base segura. Aplicaciones clínicas de la teoría de apego, Paidós, 1989]

Campbell, D. (1974). Evolutionary epistemology. En: Schilpp, P.A. (ed) The philosophy of Karl Popper. The Library of Living Philosophers, La Salle. [Epistemología Evolucionista. En: Martínez, S.; Olivé, L. Epistemología Evolucionista. Paidós, 1997]

Chiari, G.; Nuzzo, M. (1982) (Eds) Le prospettive comportamentale e cognitiva in psicoterapia. Roma: Bulzoni.

Cionini, L. (1991) Psicoterapia cognitiva: teoria e metodo dellintervento terapeutico. Roma: La Nuova Italia Scientifica.

Eкмan, P. (1972). Universal and cultural differences in facial expression of emotion. En: Cole, J.K. (Ed) Nebraska symposium on motivation 1971. Lincoln: University of Nebraska Press.

Ekman, P. (1984). Expression and nature of emotions. En Scherer, K.; EkMan, P. (Eds) Approaches to emotions. Hillsdale: Erlbaum.

Guidano, V. (1987). Complexity of the self. Guilford Press.

Guidano, V. (1991). The Self in Process. Guilford Press. [El Sí-Mismo en Proceso, Paidós, 1994] 
Guidano, V.; Liotti, G. (1979). Elementi di Psicoterapia Comportamentale. Roma: Bulzoni.

Guidano, V.; Liotti, G. (1981) Repertorio comportamentale e organizzazione comportamentale. En: Fiorelli, S; Rezzonico, G. (Eds) La modificazione del comportamento. Milano: Angeli.

Guidano, V.; Liotti, G. (1983). Cognitive Processes and Emotional Disorders. Guilford Press.

Guidano, V.; Reda, M. (1981). Cognitivismo e Psicoterapia. Milano: Angeli.

IzARD, C. (1977). Human Emotions. New York: Plenum Press.

James, W. (1890). Principles of psychology. New York: Rinehart \& Winston. [Principios de Psicología, Fondo de Cultura Económica, 1989]

Kunn, T. (1962). The structure of scientific revolutions. Chicago: University of Chicago Press. [La estructura de las revoluciones científicas, Fondo de Cultura Económica, 1971]

LAKatos, I. (1974). Falsification and the methodology of scientific research programmes. En: Lakatos, I; Musgrave, A. (Eds) Criticism and the growth of knowledge. Cambridge, UK: Cambridge University Press.

Lakatos, I. (1978). The Methodology of Scientific Research Programmes. Philosophical Papers Vol I. Cambridge, UK: Cambridge University Press. [Una Metodología de los Programas de Investigación Científica. En: La Metodología de los Programas de Investigación Científica. Ed Alianza Universidad, 1988]

Lambruschi, F. (2004). (ed) Psicoterapia cognitiva dell'età evolutive. Procedure di assessment e strategie terapeutiche. Torino: Bollati Boringhieri.

LenzI, S.; Bercelli, F. (1999). Riascoltando una seduta III, Quaderni di Psicoterapia Cognitiva 5, Vol.2, $\mathrm{n}^{\circ} 2$.

Leventhal, H. (1979). A perceptual-motor processing model of emotions. En Pliner, P.; Blankstein, K.; Spiegel, M. (Eds) Perception of emotions in self and in others. New York: Plenum Press.

Liotтi, G. (1994). La dimensione interpersonale della coscienza. Roma: La Nuova Italia Scientifica.

Liotti, G. (2006). Prólogo a la edición en español. En Guidano, V.; Liotti, G. Procesos Cognitivos y Desórdenes Mentales. Cuatrovientos.

Lorenz, K. (1973). Die Rückseite des Spiegels. München: Piper [La otra cara del espejo, Plaza \& Janés, Madrid, 1974]

Lyotard, J. (1979). La Condition postmoderne: Rapport sur le savoir [La condición postmoderna: Informe sobre el saber, Madrid, Cátedra, 1984]

Mahoney, M. (1980). Psychotherapy and the structure of personal revolutions. En Mahoney, M. Psychotherapy Process. New York: Plenum.

MaHoney, M. (1991). Human change processes: the scientific foundations of psychotherapy. New York: Basic Books.

Marmor, I. (1983). Systems thinking in psychiatry; some theoretical and clinical implications. American Journal of Psychiatry, 140, 833-838.

Maturana, H.; Varela, F. (1980). Autopoiesis and cognition: the realization of the living. Dordrecht: Reidel. 
Mead, G. (1934). Mind, self and society. Chicago: University of Chicago Press. [Espíritu, persona y sociedad, Paidós, 1972]

Meichenbaum, D. (1974). Cognitive behavior modification: an integrative approach. New York: Plenum Press.

Miller, G.; Galanter, E.; Pribram, K. (1960). Plans and the structure of behavior. New York: Holt, Rinehart \& Wilson.

Moltedo, A. (2004). Más allá de la nosografía: la visión Cognitivo Procesal Sistémica del Síntoma. Psicoperspectivas, vol III. Chile.

Moltedo, A. (2007). Le difficoltà della nosografia dei disturbi dell'umore. En NARdi B. (Ed) L'umore e I suoi disturbi. Ancona: Ed. Accademia Dei Cognitivi Della Marca.

Moltedo, A. (inédito) De las Estructuras Cognitivas a los Estilos Personales: la evolución del pensamiento de Vittorio Guidano.

Morin, E. (1977). La Méthode 1: La Nature de la Nature. Le Seuil, 1977. [El Método I: La naturaleza de la naturaleza. Cátedra. Madrid, 1981]

NArdi, B. (2005). Valenze adattive dello sviluppo delle organizzazioni di significato personale. Quaderni di Psicoterapia Cognitiva, 16 (8/1), 30-47.

Oneto, L.; Moltedo, A. (2002). Las Organizaciones de Significado Personal de Vittorio Guidano. Una llave explicativa de la experiencia humana. Psicoperspectivas, vol I, 83-91. Chile.

Panelli, G. (2003). Patologie da dipendenza ed epistemologia postrazionalista. En Nardi, B. (Ed) Normalitá e Disagio nell'Adolescenza. Ancona: Ed. Accademia Dei Cognitivi Della Marca.

Piaget, J. (1970) Psychology and epistemology: towards a theory of knowledge. New York: Viking.

Piaget, J.; Inhelder, B. (1966) La psychologie de l'enfant. Paris: Presses Universitaires de France. [Psicología del nińo. Madrid: Morata, 1981]

Polanyi, M. (1966) The tacit dimension. Garden City: Doubleday.

Popper, K. (1963). Conjectures and refutations. London: Routledge \& Kegan. [Conjeturas y refutaciones, Paidós, 1983]

Popper, K. (1972). Objective knowledge: An evolutionary approach. Oxford: Clarendon Press. [Conocimiento objetivo, Tecnos, Madrid, 1992]

Popper, K. (1974). The philosophy of Karl Popper. The Library of Living Philosophers. La Salle.

Pribram, K. (1971). Languajes of the brain: experimental paradoxes and the principles in neuropsychology. Prentice Hall, Englewood Cliffs.

Prigogine, I. (1980). From being to becoming: Time and complexity in the physical sciences. San Francisco: Freeman.

Prigogine, I. (1997). The end of certainty. New York: Free Press.

Reda, M. (1992). La relazione terapeutica: un modello strutturalista. En SACCO, G.; Isola, L. (1992) (ed) La relazione terapeutica nelle terapie cognitive. Roma: Melusina.

Rezzonico, G.; Lambruschi, F. (1996). La psicoterapia cognitiva nel servizio pubblico. Milano: FrancoAngeli. 
Ricoeur, P. (1990). Soi-même comme un autre. Paris: Èditions du Seuil. [Sí Mismo como otro, Mexico: Ed Siglo XXI, 1996]

Semerari, A. (1998). Costruttivismo e psicoterapia cognitiva. Psicobiettivo, 18, 21-38.

Semerari, A. (2000). Storia, teoria e tecniche della psicoterapia cognitiva. Roma: Laterza.

Tomkins, S. (1978). Script theory: differential magnification of affects. En Howe, H.; PAge, M. (Eds) Nebraska Symposium on motivation 1978. Lincoln: University of Nebraska Press.

Vattimo, G. (1983). Il pensiero debole. Milano: Feltrinelli. [Pensamiento débil. Madrid: Cátedra, 3ra edición, 1995]

Von Bertalanffy, L. (1968). General system theory. London: Lane. [Teoría general de los sistemas: fundamentos, desarrollo, aplicaciones. Ciudad de México: Fondo de Cultura Económica, 2da edición 1991]

Von Foerster, H. (1981). Observing systems. Intersystems Publications, Seaside. [Sistemi che osservano. Roma: Astrolabio, 1987]

Von Glaserfeld, E. (1984). An introduction to radical constructivism. En WatzLAWICK, P. (ed) The invented reality. New York: Norton. [La realidad inventada. Barcelona: Gedisa, 1989.]

Von Hayek, F. (1952). The sensory order. The University of Chicago Press.

Weimer, W. (1977). A conceptual framework for cognitive psychology: Motor theories of the mind. [Un sistema di riferimento concettuale per la psicologia cognitivista: le teorie motorie della mente. En: CARAMELLI, N. (Ed) La psicologia cognitivista. Bologna: Il Mulino, 1983].

WIENER, N. (1948). Cybernetics, or control and communication in the animal and machine. New York: Wiley. [Cibernética. Barcelona: Tusquets Editores, 1985].

Zagmutt, A. (2006). La evolución de la Terapia Cognitiva Posracionalista. Conferencia plenaria ofrecida durante el VI Congreso Latinoamericano de Terapias Cognitivas, Buenos Aires, Mayo 2006.

Fecha de Recepción de artículo: 18 de Julio 2008

Fecha de Aceptación de artículo: 05 de Septiembre 2008 
